\title{
Característica Clínico-epidemiológica de los pacientes pediátricos con Cetoacidosis Diabética
}

\author{
Clinical and epidemiological characteristics of pediatric patients with diabetic ketoacidosis
}

\author{
Sofía Alejandra Gutiérrez,* Oscar Francisco Zúniga Girón.**
}

\begin{abstract}
RESUMEN
Antecedentes: La Cetoacidosis diabética (CAD) es una complicación de la Diabetes Mellitus, cuya importancia va cada vez en aumento. Es el trastorno metabólico que causa más ingresos a las unidades de cuidado intensivo pediátrico con una tasa de hospitalización que se ha mantenido alrededor de 10 por 100.000 niños/año. Objetivo: Describir las características clínico-epidemiológicas de la cetoacidosis diabética en los pacientes menores de 18 años. Pacientes y métodos: Se realizó un estudio descriptivo. La información fue recopilada mediante encuesta sobre características socio-demográficas, epidemiológicas y diagnóstica en niños con CAD que fueron ingresados en la emergencia de pediatría del Hospital Nacional Dr. Mario Catarino Rivas (HNMCR), durante el período de agosto 2013 a agosto del $2015(n=30)$. Resultados: Se encontró 18 (60\%) mujeres y 12 (40\%) varones con CAD. El promedio de edad de los pacientes diagnosticados con CAD fue de 12 años, con una mediana de 13 años. El 50\% de los pacientes con CAD eran debutantes y el $20 \%$ tenían 2 años de diagnóstico. Las infecciones de tracto urinario estuvieron presentes en el $40 \%$ de los casos. El promedio de glicemia sérica en los pacientes con CAD fue de $500 \mathrm{mg} / \mathrm{dl}$. El edema cerebral fue la principal complicación encontrada en un $33.3 \%$ de los casos. Fallecieron el 7\% de los pacientes con CAD. Conclusiones: La CAD es frecuente en mujeres adolescentes $y$ es desencadenado principalmente por infecciones del tracto urinario.
\end{abstract}

Residente de 3er. año del Posgrado de Pediatría Universidad Nacional Autónoma de Honduras en el Valle de Sula (UNAHVS) Endocrinólogo Pediátra Dr. Mario Catarino Rivas (HNMCR) Dirigir correspondencia a: sophy0319@hotmail.com Recibido: 15 de octubre 2015 Aprobado: 19 de enero 2016

\author{
PALABRA CLAVE \\ Cetoacidosis Diabética, Diabetes Mellitus, \\ hiperglicemia.
}

\begin{abstract}
Background: Diabetic ketoacidosis (DKA) is a complication of Diabetes Mellitus, whose importance is ever increasing. It is the metabolic disorder that causes more admissions to the pediatric intensive care units, with hospitalization rates around 10 per 100,000 children per year. Objective: To describe the clinical and epidemiological characteristics of diabetic ketoacidosis in patients under 18 years.
\end{abstract} Patients and methods: A descriptive study was conducted. The information was collected through a survey on socio-demographics, epidemiology and diagnosis in children with DKA who were admitted to the pediatric emergency at the National Hospital Dr. Mario Catarino Rivas (HNMCR) during the period August 2013 to August $2015(n=30)$. Results: $18(60 \%)$ women and 12 (40\%) men with DKA were found. The average age at which patients were diagnosed with DKA was 12 years, with a median of 13 years. $50 \%$ of patients with DKA were newly onset and 20\% were diagnosed 2 years before. Urinary tract infections were present in $40 \%$ of cases. The average of serum glucose in patients with DKA was $500 \mathrm{mg} / \mathrm{dl}$. The cerebral edema was the main complication found in $33.3 \%$ of cases. $7 \%$ of patients with DKA died. Conclusions: DKA is the most common complication of diabetes with higher incidence in adolescent girls and it is triggered primarily by "urinary tract infections".

\section{KEYWORDS}

Diabetic ketoacidosis, diabetes mellitus, hyperglycemia 


\section{INTRODUCCIÓN}

La Diabetes mellitus infantil es una de las patologías crónicas más frecuentes de la edad pediátrica. La incidencia anual de la CAD varía de 4,6 a 8 episodios por 1.000 pacientes con diabetes y es la principal causa de morbilidad y mortalidad en casos de diabetes mellitus tipo $1 .^{(1)}$ Se caracteriza por la existencia de hiperglucemia en ayunas y post ingesta, y es secundaria al déficit absoluto de la insulina. La diabetes no es una enfermedad única, sino que engloba a un grupo heterogéneo de alteraciones del metabolismo de los hidratos de carbono con distinto patrón genético, así como diferentes etiologías y mecanismos fisiopatológicos. ${ }^{(2)}$

La complicación aguda más frecuente en niños con Diabetes Mellitus tipo 1 es la Cetoacidosis Diabética (CAD); se debe a una descomposición metabólica grave manifestada por la sobreproducción de cuerpos cetónicos y cetócidos que se desplazan al torrente sanguíneo y resultan en cetoacidosis metabólica. ${ }^{(2)}$

La cetosis y la acidosis contribuyen a producir alteraciones hidroelectrolíticas, vómitos y deshidratación. La hiperglucemia puede ser relativamente leve, (no tan pronunciada como el coma hipersomolar), y está asociada también con cetonemia. La CAD es la complicación aguda que requiere más hospitalizaciones $(15 \%)$ y más ingresos a la unidad de cuidados intensivos (10\%). Los principales factores generadores y desencadenantes de la CAD; son la neumonía y la infección urinaria (30\% a 40\% de los casos) seguidas por gastroenteritis e infecciones del tracto respiratorio superior. ${ }^{(3)}$ El tratamiento de la CAD es una emergencia y se basa en la administración de líquidos, restablecer el déficit de electrolitos, revertir la acidosis, la deshidratación y reducir la hiperglicemia. La mayoría de las muertes por CAD son prevenibles con un buen manejo inicial: reposición gradual de líquidos, adición oportuna de dextrosa a la hidratación y terapia con insulina. ${ }^{(4)}$

La mortalidad predominantemente se asocia al edema cerebral y sólo una minoría de las muer- tes se atribuye a otras causas. El edema cerebral ocurre en cerca de un $0,3-1 \%$ de todos los episodios de CAD, pero causa el 57 al $87 \%$ de las muertes por CAD. ${ }^{(5-6)}$ Otras causa posibles de mortalidad y morbilidad incluyen hiperpotasemia, hipoglucemia, complicaciones en el sistema nervioso central (SNC), como hematomas, trombosis, sepsis y edema pulmonar, entre otras. Las secuelas tardías asociadas a edema cerebral y otras complicaciones del SNC incluyen la insuficiencia hipotálamo-hipofisaria, la deficiencia de somatotropina $(\mathrm{GH})$ y la deficiencia combinada de $\mathrm{GH}$ y tirotropina (TSH). ${ }^{(6)}$

En Honduras representa el 85\% de las patologías endocrinológicas en el adulto, la 11va causa de ingresos en los hospitales públicos, y la 14va causa de mortalidad; en el área pediátrica se cuenta con pocos estudios sobre esta patología. ${ }^{(7)}$ Existen pocos datos estadísticos sobre la situación epidemiológica actual de la CAD en pediatría en la zona norte del país, particularmente áreas de influencia del HNMCR, por lo que se decide realizar este estudio con el fin de identificar la forma de presentación clínica más frecuente de los niños CAD y las características epidemiológicas de la población en estudio.

\section{PACIENTES Y MÉTODOS}

Se realizó un estudio descriptivo, observacional, transversal y con un componente prospectivo. La población en estudio fue conformada por niños ambos sexos menores de 18 años que acudieron a la emergencia de pediatría del HNMCR, durante el período de agosto 2013 a agosto del 2015, con el diagnóstico de Cetoacidosis Diabética. El diagnóstico se realizó al presentar la clínica y hallazgos laboratoriales de CAD que son los siguientes: hiperglucemia (glucosa en sangre (BG) $>11 \mathrm{mmol} / \mathrm{L}(200 \mathrm{mg} / \mathrm{dl})$, $\mathrm{Ph}<7.3$ y bicarbonato $<15 \mathrm{mmol} / \mathrm{L}$, cetonemia y cetonuria. Los signos clínicos de la CAD incluyen: la deshidratación, taquicardia, taquipnea (respiración de Kussmaul), aliento cetónico (olor a acetona o fruta podrida), náuseas, vómitos, dolor abdominal, confusión, somnolencia, reducción progresiva del nivel de conciencia y la pérdida de conciencia. ${ }^{(8)}$ 
Se excluyó del estudio los pacientes diabéticos con pancreatitis, con crisis de asma, acidosis respiratoria, con trauma, con quemadura, y con fibrosis quística, o si no se obtuvieron los datos completos de los expedientes. El instrumento constó de 20 preguntas cerradas, fue llenado por el médico residente a cargo de la investigación, a través de la revisión de expedientes clínicos con aprobación de las autoridades del hospital. Los datos obtenidos fueron: datos sociodemográficos (edad, género y procedencia), antecedentes de DM, tiempo de diagnóstico, signos y síntomas de presentación, factores desencadenantes, estadía hospitalaria. Se registró el método diagnóstico clínico y laboratorial (gases arteriales, electrólitos séricos, setonuria). Se revisaron 45 expedientes con pacientes con CAD que cumplieron los criterios de inclusión, pero se excluyeron 15 que no tenían exámenes de laboratorio. Se captaron un total de 30 pacientes.

Los datos obtenidos fueron tabulados mediante el programa estadístico SPS 22.0 y Microsoft Excel 2010. Para el análisis de datos se utilizaron medidas de tendencia central (promedio, moda y mediana), frecuencia (porcentaje), medidas de dispersión (desviación estandar).

\section{RESULTADOS}

En la tabla No. 1 se presentan los resultados sobre las características socio demográficas de la población en estudio. El promedio de edad de los pacientes diagnosticados con CAD fue de 12 años, con una mediana de 13 años con la edad moda de 14 años. El $60 \%$ de los pacientes con diagnóstico de CAD que acudieron al servicio de emergencia de pediatría corresponden al sexo femenino.

El 50\% de los pacientes residen en el área rural. Los pacientes con CAD procedían del departamento de Cortes (60\%), y del departamento de Yoro (20\%).

En la tabla No. 2 podrá encontrar información relevante sobre la historia del paciente con CAD. El $50 \%$ de los pacientes con CAD eran debutantes para diabetes mellitus. Y el $20 \%$ tenían 2 años de diagnóstico. Las infecciones del tracto urinario desencadenaron el $68 \%$ de las CAD $16.6 \%$ por abandono del tratamiento, el $16.5 \%$ por uso de dosis inadecuadas de insulina y el $6.7 \%$ por trastornos en la dieta.

Tabla No. 1: Características socio-Demográficas de pacientes con CAD.

\begin{tabular}{cc}
\hline $\begin{array}{c}\text { Características Socio- } \\
\text { Demográficas }\end{array}$ & $\mathbf{n ( \% )}$ \\
\hline Edad (promedio (DE†) & $12(3-16)$ \\
$1-3 a n ̃ o s$ & $12(40 \%)$ \\
$4-7$ años & $1(3.3 \%)$ \\
$8-10$ años & $2(6.6 \%)$ \\
Género Femenino. & $18(60 \%)$ \\
Procedencia & $15(50 \%)$ \\
Rural & $18(60 \%)$ \\
Cortés & $6(20 \%)$ \\
Yoro & $2(6.7 \%)$ \\
Santa Bárbara & $1(3.3 \%)$ \\
Colón & $1(3.3 \%)$ \\
Comayagua &
\end{tabular}

†DE: Desviación estándar

Fuente: Instrumento características clínico-epidemiológicas en la CAD

Los signos y síntomas reportados en los pacientes con CAD evaluados de acuerdo al orden de frecuencia en esta investigación fueron: la poliuria (76.7\%), polidipsia (73.3\%), deshidratación $(66.7 \%)$, pérdida de peso $(56.5 \%)$, dolor abdominal (53.3\%), anorexia (50\%) y respiración kussmaull (36\%).

El inicio de síntomas previa atención médica de los pacientes con CAD fue de un rango de 1-5 días (57\%), y solo un 30\% los presentó entre 6-10 días. (Ver tabla No. 2).

Al momento de su ingreso al hospital el $77 \%$ tenía una escala de Glasgow de 13-15 puntos y el $7 \%$ presentó una puntuación menor de 9 
puntos. (Ver tabla No. 3). El promedio de glicemia sérica en los pacientes con CAD fue de 500 $\mathrm{mg} / \mathrm{dl}$ al igual que la moda. Con un rango entre $315 \mathrm{mg} / \mathrm{dl}$ a $680 \mathrm{mg} / \mathrm{dl}$. La mayoría de los pacientes con CAD presentó acidemia severa (57\%). El promedio de los electrolitos en los pacientes con CAD de sodio, Potasio y cloro se encontró dentro de parámetros normales, con una osmolaridad promedio de $310 \mathrm{mOsm} / \mathrm{L}$.

Todos los niños con CAD que ingresaron en el estudio presentaron cetonuria +++ .

Tabla No. 2: Historia clínica en los pacientes con CAD.

\begin{tabular}{cc}
\hline Historia clínica & $\mathbf{n ( \% )}$ \\
\hline $\begin{array}{c}\text { Tiempo de diagnóstico DM } \\
\text { Debutante } \\
\text { Diagnóstico previo } \\
1 \text { año }\end{array}$ & $15(50 \%)$ \\
2 años & $2(7 \%)$ \\
3 años & $6(20 \%)$ \\
5 años & $4(14 \%)$ \\
9 años & $1(3 \%)$ \\
10 años & $1(3 \%)$ \\
Inicio de síntomas previo al ingreso \\
1-5 días & $17(5 \%)$ \\
6-10 días & $9(30 \%)$ \\
Mayor de 10 días. & $4(13 \%)$ \\
\hline
\end{tabular}

†DM: Diabetes Mellitus

Fuente: Instrumento características clínico-epidemiológicas en la CAD

Entre las complicaciones de la CAD, se encontró el choque hipovolémico en un $16.6 \%$, edema cerebral en un $10 \%$ e insuficiencia pre-renal en un $6.6 \%$.

La mitad de los pacientes permaneció en el hospital por más de 10 días ( $n=15)$, mientras un $36.7 \%(n=11)$ presentó una estadía intrahospitalaria de $6-10$ días, y el $16.6 \%$ restante $(n=5)$ entre 1 a 5 días.

Las recaídas de los pacientes con CAD en nuestro estudio representaron el $30 \%$ de los casos.
Se encontró que 17 (57\%) pacientes tenian acidemia severa y 10 (33\%) teniam acidemia moderada. (Ver tabla No. 3)

Tabla No. 3: Hallazgos Clínico-Laboratoriales en pacientes con CAD.

\begin{tabular}{lc}
\hline \multicolumn{1}{c}{$\begin{array}{c}\text { Hallazgos Clínico } \\
\text { laboratoriales }\end{array}$} & $\mathbf{n ( \% )}$ \\
\hline $\begin{array}{l}\text { Glasgow al ingreso } \\
\text { (puntuación 13-15) }\end{array}$ & $23(77 \%)$ \\
Puntuación 9-12 & $5(16 \%)$ \\
Puntuación <9 & $2(7 \%)$ \\
Glicemia al ingreso (x* & $500(315-680)$ \\
(DE †)) (mg/dL) & \\
Acidemia & \\
$\quad$ severa (pH <7.1) & $17(57 \%)$ \\
$\quad$ moderada (pH 7.1- 7.2) & $10(33 \%)$ \\
$\quad$ leve (pH 7.2 -7.3) & $2(7 \%)$ \\
$\quad$ no consignado & $1(3 \%)$ \\
Sodio (x; mg/dL) & 139 \\
Potasio (x; mg/dL) & 4.4 \\
Cloro (x; mg/dL) & 109 \\
\hline
\end{tabular}

†DE: desviación estándar, ${ }^{*} x$ : promedio

Fuente: Instrumento características clínico-epidemiológicas en la CAD

Dos pacientes con diagnóstico CAD fallecieron. El rango de edad de fallecidos por CAD corresponde entre 6-15 años. La tasa de letalidad de los pacientes con CAD fue de un $6.6 \%$.

(Ver tabla No.4)

Tabla No. 4: Tasa de letalidad en los Pacientes con CAD.

\begin{tabular}{lccc}
\hline Sexo & $\begin{array}{c}\text { Número } \\
\text { de casos }\end{array}$ & $\begin{array}{c}\text { Número de } \\
\text { fallecidos }\end{array}$ & $\begin{array}{c}\text { Letalidad } \\
\text { (\%) }\end{array}$ \\
\hline Mujer & 18 & 1 & 8.3 \\
Hombre & 12 & 1 & 5.6 \\
Total & 30 & 2 & 6.6
\end{tabular}

Fuente: Instrumento características clínico-epidemiológicas en la CAD 


\section{DISCUSIÓN}

La estadística sobre la presentación de la CAD es muy variable en la literatura. Los resultados concuerdan con estudios realizados por Martínez García y col. en México en el 2009, ${ }^{(6)}$ donde la CAD se presentó en mujeres (60\%), y la edad promedio de los pacientes fue de 12 años (2-16 años en el HNMCR y 3-16 años en México). Sin embargo, difiere a resultados obtenidos en Colombia (2010) donde la edad promedio fue de 8,75 años siendo más baja que la encontrada en esta investigación. ${ }^{(9)}$

En el HNMCR no se encontró diferencia entre la procedencia de zona rural y zona urbana, (50\% respectivamente). Sin embargo en investigaciones realizadas por Discua Flores y colaboradores $^{(10)}$ en Tegucigalpa y Ballesteros ${ }^{(9)}$ en Colombia respectivamente, encontraron que la mayoría de los pacientes provenían de la zona urbana en un $86.6 \%$. El 50\% presentaron antecedentes familiares de primer y segundo grado de consanguinidad para diabetes, correlacionándose con el estudio realizado en México en el año 2009 , donde el $43 \%$ de los casos presentaba antecedentes de diabetes familiar. ${ }^{(6)}$

En esta investigación la mitad de los pacientes eran debutantes al igual que en investigaciones realizadas en Colombia (2010), a diferencia del estudio realizado por Discua Flores 2006, en el cual el $36.7 \%$ fueron debutantes. ${ }^{(9)}$

Se encontró que los signos y síntomas en esta investigación son similares a los encontrados en la literatura siendo así la poliuria, polidipsia y deshidratación. En un estudio realizado por Carlotti (2009) el signo más frecuente fue la poliuria y los vómitos. ${ }^{(11)}$

Los factores desencadenantes de mayor relevancia encontrados fueron las infecciones, específicamente las infecciones urinarias, seguido de la no aplicación de insulina y el abandono de tratamiento. Sin embargo en el estudio realizado en Honduras en el año 2006 las infecciones más frecuentes fueron las respiratorias $(56.6 \%)$. ${ }^{(9)}$
En relación al inicio de los síntomas previa atención médica en este estudio se encontró una mediana de 5 días, correlacionándose con el estudio realizado en Honduras en donde la mitad de los pacientes asistió al hospital antes de los 5 días de iniciado los síntomas de CAD. ${ }^{(9)}$ Los días intrahospitalario de los pacientes con CAD en este estudio fue mayor de 10 días al igual que en los tres estudios antes mencionados.

En cuanto al nivel de conciencia de los pacientes con CAD en este estudio el 25\% tenían una escala de Glasgow menor 12 puntos. Según la literatura los pacientes presentan algún tipo de déficit neurológico evidenciado por el puntaje menor de 15 en la escala de Glasgow. ${ }^{(12)} \mathrm{El}$ promedio de glicemia sérica en los pacientes con Cetoacidosis Diabética fue de 500 mg/dl comparándose con varios estudios en donde la glucemia al ingreso fue de $428 \mathrm{mg} / \mathrm{dL}$. $^{(10)}$

La mayoría de los pacientes con CAD presentó en el primer análisis gasométrico acidemia severa (57\%), moderada (33\%), leve (7\%) y no consignada (3\%).

En relación al equilibrio hidroelectrolítico en este estudio se contó unicamente los niveles de electrolitos los cuales estaban dentro de los rangos normales, sin embargo no se descarta que existieran alteraciones electrolíticas durante el manejo de los pacientes con CAD, ya que solamente se realizó una medición. La literatura reporta alteraciones como hipokalemia, hipocalcemia e hiponatremia. ${ }^{(11)}$

El choque hipovolémico y el edema cerebral fueron las complicaciónes de mayor importancia respectivamente; en un estudio de Wolfsdorg (2006), reportaron que el edema cerebral se presentó en $46.6 \%$ de los pacientes. ${ }^{(12)} \mathrm{La}$ frecuencia de edema cerebral en niños con CAD ha sido reportada alrededor del 1\% al 3\% en el mundo, ${ }^{(13-17)}$ pero en este estudio fue del $10 \%$ lo cual puede estar relacionado con el mayor porcentaje de cetoacidosis grave al ingreso. En esta investigación fallecieron 2 pacientes del sexo femenino y masculino con edades de 6 y 14 años y con una tasa de letali- 
dad de $6.6 \%$ siendo más alto este porcentaje que el encontrado en el estudio realizado en el Hospital Escuela de Honduras, entre 2004 y 2006. ${ }^{(10)}$

Cabe mencionar que este estudio tiene un universo de casos limitado a 30 durante el periodo de observación. Esto limita conclusiones estadísticas. Se espera que se vuelva a replicar el estudio para aumentar casuística para obtener un reflejo más fidedigno de datos. Sin embargo, es evidente que el país requiere un protocolo de manejo de los casos de CAD en menores de 18 años enfocado en las actividades de detección, diagnóstico, tratamiento que pueda ser aplicado por médicos generales y médicos en servicio social formativo en áreas regionales. Se debe de garantizar la disponibilidad de pruebas básicas para el diagnóstico de $C A D$, como son la gasometría arterial, electrolitos séricos, tiras reactivas, para así poder ofrecer la posibilidad de un seguimiento oportuno.

En conclusión la CAD es frecuente en mujeres adolescentes desencadenado principalmente por infecciones del tracto urinario.

\section{BIBLIOGRAFÍA}

1. Kitabchi AE, Umpierrez GE, Murphy MB, Kreisberg RA. Hyperglycemic crises in adult patients with diabetes: a consensus statement from the American Diabetes Association. Diabetes Care. [Revista en internet] 2006 [citado 20 de marzo 2015]. 29: 27392748. Disponible en http//. care care.diabe tesjournals.org.

2. Céspedes Camila, Bustos Carolina. Manejo de la cetoacidosis diabética en niños y adolescentes Endocrinol Nutr. [Revista en internet] 2008 [Citado 21 octubre 2015]; 55(7):289-96. Disponible en: http://www. elsevier.es/es-revista-endocrinologia-nutri cion-12-articulo-manejo-cetoacidosis-dia betica-ninos-adolescentes-13125710

3. Hanas R, Lindgren F, Lindblad B. A 2-year National Population Study of Pediatric ketoacidosis in Sweden: predisposing conditions and insulin pump use. Pediatr Diabetes [Revista en internet], 2006 [citado 20 marzo 2015], 29 (5): 1150-1159. Disponible en: http://www. ncbi.nlm.nih.gov/pub med/18761647.

4. Cox K, Cocchi MN, Salciccioli JD, Carney E, Howell M, Donnino MW. Prevalence and significance of lactic acidosis in diabetic ketoacidosis. J Crit Care [Revista en inter- net]. 2012 [citado 19 marzo 2014]; 27: 132-137. Disponible en: http://www. ncbi. nlm.nih.gov/pubmed/22033060.

5. Deeter KH, Roberts JS, Bradford H, Richards T, Shaw D, Marro K, et. al. Hypertension despite dehydration during severe pediatric diabetic ketoacidosis. Pediatr Diabetes [Revista en internet]. 2011 [citado 22 marzo 2014]; 12 (4 Pt 1): 295-301. Disponibleen: http://www.ncbi.nlm.nih.gov/pmc/articles /PMC3103609/.

6. Martínez García JJ, Torres-Galicia CG, Gastélum-Benítez JE. Características clínicas de niños con cetoacidosis diabética. Paediatr México [Revista Médica] 2009; 2(2): 55-69. [citado 28 mayo 2014]Disponible en: http://paediatria.com/archivo/pa ediatr-mex-2009-vol-2-no-2/caracteristicas -clinicas-de-ninos-con-cetoacidosis-diabe tica/

7. McDonnell CM, Pedreira CC, Vadamalayan B, Cameron FJ, Werther GA. Diabetic ketoacidosis, hyperosmolarity and hypernatremia: are high-carbohydrate drinks worsening initial presentation? Pediatr Diabetes. [Internet] 2005[Citado 13 de octubre 2015] Jun;6(2):90-4. Disponible en: http://www. ncbi.nlm.nih.gov/pubmed/15963036 
8. Jiménez Fadul Ana M, Cortés Millán JC. Enfoque diagnóstico y terapéutico de la cetoacidosis diabética en niños y adolescentes en el servicio de urgencia niños y adolescentes en el servicio de urgencias. latreia [Revista en internet]. julio-septiembre, 2013 [citado 28 mayo 2014]; 26(3):325-335. Disponible en: http://www. redalyc.org/pdf/1805/180528412008.pdf

9. Ballesteros Calderón AL, Meneses Muñoz AP, Patiño DR, Flórez Gómez ID, Quevedo Vélez A. Características epidemiológicas y clínicas y hallazgos de laboratorio de los niños menores de 15 años con Cetoacidosis Diabética atendidos en el Hospital Universitario San Vicente Fundación en Medellín, Colombia, entre enero de 2001 y diciembre de 2010. latreia [Revista en internet]. julio-septiembre, 2013[citado 10 Julio 2015]; 26(3): 278-290. Disponible en: http://www.redalyc.org/articulo.oa?id= 180528412004

10. Discua Flores LJ, Villatoro G, Aguilera R. Perfil epidemiológico y clínico de la cetoa cidosis diabética en los pacientes que asisten al servicio de pediatría del Hospital Escuela, julio 2004 a junio 2006. Revista Médica de los Post Grados de Medicina UNAH [Revista en internet]. 2007[Citado 10 julio 2015]; 10(3):200-204. Disponible en: http://cidbimena.desastres.hn/RMP/pdf/ 2007/pdf/Vol10-3-2007-9.pdf

11. Carlotti AP, St George-Hyslop C, Guerguerian AM, Bohn D, Kamel KS, Halperin MI. Occult risk factor for the development of cerebral edema in children with diabetic ketoacidosis: possible role for stomach emptying. Pediatr Diabetes [Revista en internet]. 2009[citado Agosto 2015]; 10(8): 522-533. Disponible en: http://www.ncbi. nlm.nih.gov/pubmed/19460121

12. Wolfsdorg J, Glaser N, Sperling MA. Diabetic Ketoacidosis in Infants, childrens and ado- lescents. A consensus statement fron the American Diabetes Association. Diabetes Care.2006; 29(5):1150-1159.DOI: 10.2337/dc06-9909

13. Butler A, Talbot N, Burnett C, Stanbury J, MacLachlan E. Metabolic studies in diabetic coma. Trans Assoc Am Physicians. [Revista internet]. 2006 [citado junio 2014]; 60: 102-109. Disponible en: http://www.ncbi. nlm.nih.gov/pubmed/18917248

14. Danowski, T. S., Peters, J. H., Rathbun, J. C., Quashnock, J. M., \& Greenman, L. Studies in diabetic acidosis and coma, with particular emphasis on the retention of administered potassium. Journal of Clinical Investigation. [Internet] 1949[Citado 15 de junio 2014];28(1): 1-9.Disponible en: http://www. ncbi.nlm.nih.gov/pmc/articles/PMC 439567/

15. Dunger DB, Sperling MA, Acerini CL, Bohn DJ, Daneman D, Danne TP, et. Al. ESPE/LWPES consensus statement on diabetic ketoacidosis in children and adolescents. Arch Dis Child [Revista Internet]. 2004 [citado13 junio2014]; 89(2): 188-194. Disponible en: http://www.ncbi.nlm.nih. gov/pubmed/14736641

16. Sheikh-Ali M, Karon BS, Basu A. Can serum beta-hydroxybutyrate be used to diagnose diabetic ketoacidosis? Diabetes Care [Revista Internet]. 2008 [Citado 16 Mayo 2014]; 31: 643-647. Disponible http// ncbi. nlm.nih.gov.

17. Fazeli Farsani $S$, van der Aa MP, van der Vorst MM, Knibbe CA, de Boer A. Global trends in the incidence and prevalence of type 2 diabetes in children and adolescents: a systematic review and evaluation of methodological approaches. Diabetología [Revista Internet] 2013 [Citado 12 Abril 2015]; 56(7): 1471-1488. Disponible en: http://www.ncbi.nlm.nih.gov/pubmed/ 23677041 